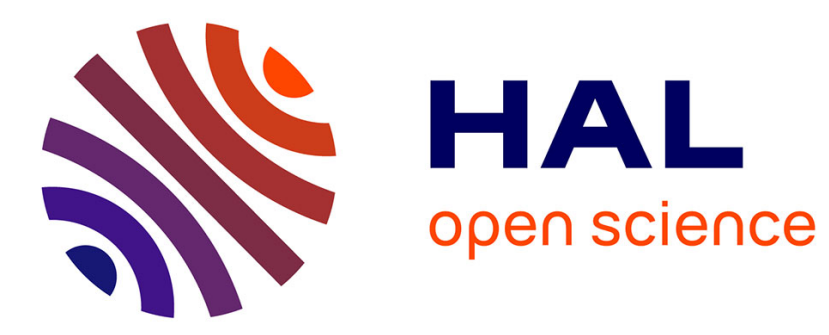

\title{
The dynamics of polar jet streams as depicted by the METEOSAT WV channel radiance field
}

D. Ramond, H. Corbin, Desbois M., Szejwach J., P. Waldteufel

\section{To cite this version:}

D. Ramond, H. Corbin, Desbois M., Szejwach J., P. Waldteufel. The dynamics of polar jet streams as depicted by the METEOSAT WV channel radiance field. Monthly Weather Review, 1981. hal01945686

\section{HAL Id: hal-01945686 \\ https://hal.uca.fr/hal-01945686}

Submitted on 8 Jun 2021

HAL is a multi-disciplinary open access archive for the deposit and dissemination of scientific research documents, whether they are published or not. The documents may come from teaching and research institutions in France or abroad, or from public or private research centers.
L'archive ouverte pluridisciplinaire HAL, est destinée au dépôt et à la diffusion de documents scientifiques de niveau recherche, publiés ou non, émanant des établissements d'enseignement et de recherche français ou étrangers, des laboratoires publics ou privés. 


\title{
The Dynamics of Polar Jet Streams as Depicted by the METEOSAT WV Channel Radiance Field
}

\author{
D. Ramond and H. Corbin \\ Observatoire du Puy de Dôme, 63000 Clermont-Ferrand, France \\ M. Des̄boIs and G. SzejWach \\ Laboratoire de Météorologie Dynamique du CNRS, Ecole Polytechnique, 91128 Palaiseau, France \\ P. WALDTEUFEL \\ Observatoire du Puy de Dôme, 63000 Clermont-Ferrand, France.
}

(Manuscript received 14 November 1980, in final form 2 June 1981)

\section{ABSTRACT}

\begin{abstract}
A polar jet stream flowing over northern Europe is investigated using both the radiosounding network and the METEOSAT water vapor (WV) channel imagery. A maximum radiance band associated with the polar jet stream is shown to accurately delineate the tropopause break; a major difference in the subtropical jet stream radiative signature is noted, however. An analytic approximation of the radiative transfer equation is established for the METEOSAT WV channel and leads to the conclusion that the radiance field, away from the polar frontal zones, is representative of the temperature field on a water vapor isosteric surface.
\end{abstract}

\section{Introduction}

Since the first satellite measurements in the 6.3 $\mu \mathrm{m}$ water vapor vibration-rotation band were obtained, a number of studies have been devoted to the relationship between the upper tropospheric dynamics and the radiance field in this spectral band. It has been noted earlier that the jet streams, which constitute a major feature of upper tropospheric dynamics, were commonly associated with a dark (warm) band running along their axis on their cyclonic side (Nordberg et al., 1966). Such a typical pattern of the radiance field was qualitatively explained as the result of the subsiding motion taking place in the vicinity of the jet streaks. In a case study, Rodgers et al. (1976) compared Nimbus 4 THIR $6.7 \mu \mathrm{m}$ data to the outputs of a 10-level diagnostic model and confirmed in a quantitative manner that the water vapor radiometric data were indicative of three-dimensional advection processes in cloud-free areas.

Studying 60 cases of subtropical jet streams, Martin and Salomonson (1970) showed the typical radiative pattern associated with the jet streaks to be so well defined that they were able to derive accurate polynomial relationships between the wind-speed maximum value and the radiative temperatures measured at grid points surrounding its location. However, these results do not seem to apply to polar jet streams. A day-to-day examination, even superficially, of the METEOSAT WV channel imagery and the $\mathbf{3 0 0} \mathrm{mb}$ or maximum wind analysis chart shows that if indeed the polar jet stream is marked by a dark (warm) band on its cyclonic side, the quantitative distribution of the radiance amplitude along this band apparently is uncorrelated with the locations of the jet streaks. Thus, it has appeared useful to study in more detail the relationship between the radiance and the dynamic fields in the polar jet stream areas where a large amount of the stratosphere-troposphere exchanges actually take place. This is the main purpose of the present paper, where in a case study of a polar jet stream, the METEOSAT 5.7-7.4 $\mu \mathrm{m}$ channel data are compared with the results of a three-dimensional subjective isentropic analysis. A further implicit purpose is to illustrate how METEOSAT, in spite of its geostationary location, remains a highly valuable tool for dynamic studies even at high latitudes (up to $68^{\circ} \mathrm{N}$ in this case study).

\section{A case study, 14 July 1978}

On 14 July 1978, a trough developed over northern Europe and led to a sudden cold-air out- 


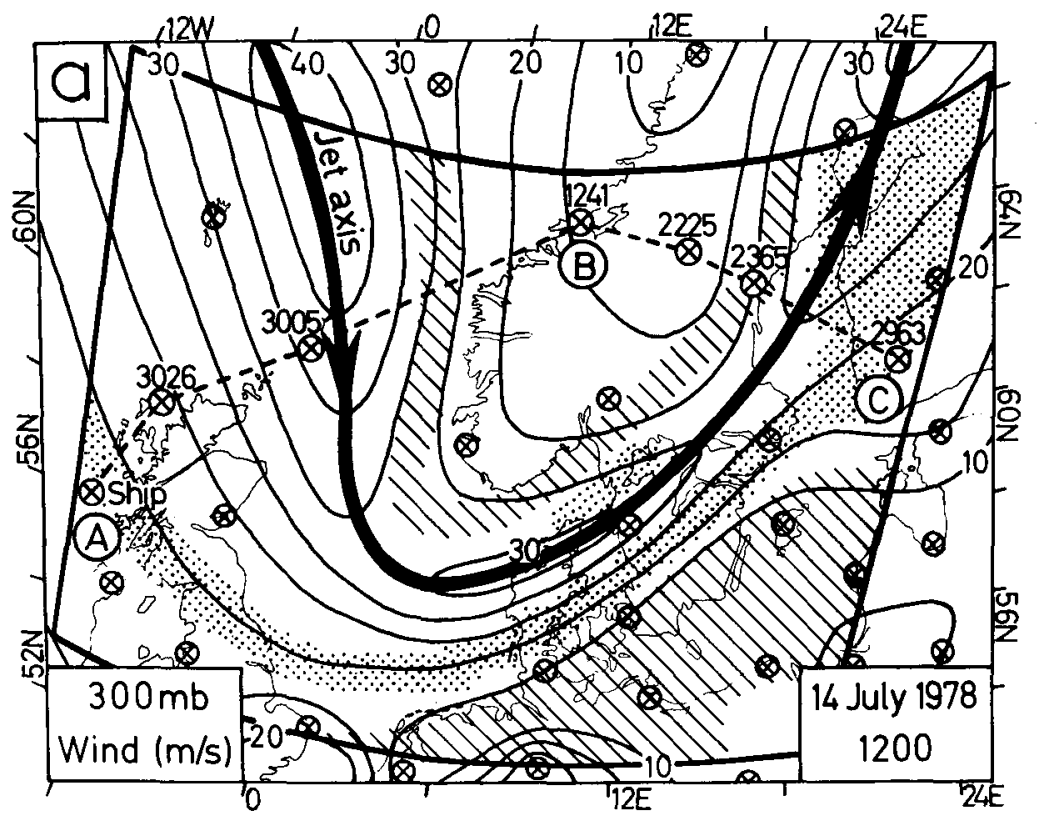

FIG. 1a. $300 \mathrm{mb}$ level isotachs $\left(\mathrm{m} \mathrm{s}^{-1}\right)$ over northern Europe $1200 \mathrm{GMT} 14$ July 1978 UTM projection. Dark bands of the simultaneous METEOSAT WV image are schematically represented by dashed areas, and cirrus cover by dotted areas. Circled crosses are for radiosounding station locations. The curvilinear rectangle marks the boundaries of the area covered by Fig. $1 \mathrm{~b}$.

break over central and southeastern Europe during the next day. At 1200 GMT the polar jet stream is seen to be associated with a well-defined dark band on the METEOSAT WV channel image (Fig. 1).

Martin and Salomonson (1970) have shown that the radiance field in the $6 \mu \mathrm{m}$ band in the vicinity of subtropical jet streaks was consistent with the deductions made by Reiter (1963, pp. 136 and 355) from his "advective jet model", i.e., high equivalent blackbody temperature $\left(T_{B B}\right)$ zones are correlated with expected convergence (subsiding motion) zones at jet stream level, and low $T_{B B}$ zones with divergence (rising motion) zones. In the present study, however, while the dark band, as expected, is on the cyclonic side of the jet axis, the distribution of the measured radiance along the band is quite different from what would be deduced from the advective jet model. Indeed, the radiance maximum is located on the southern edge of the trough,

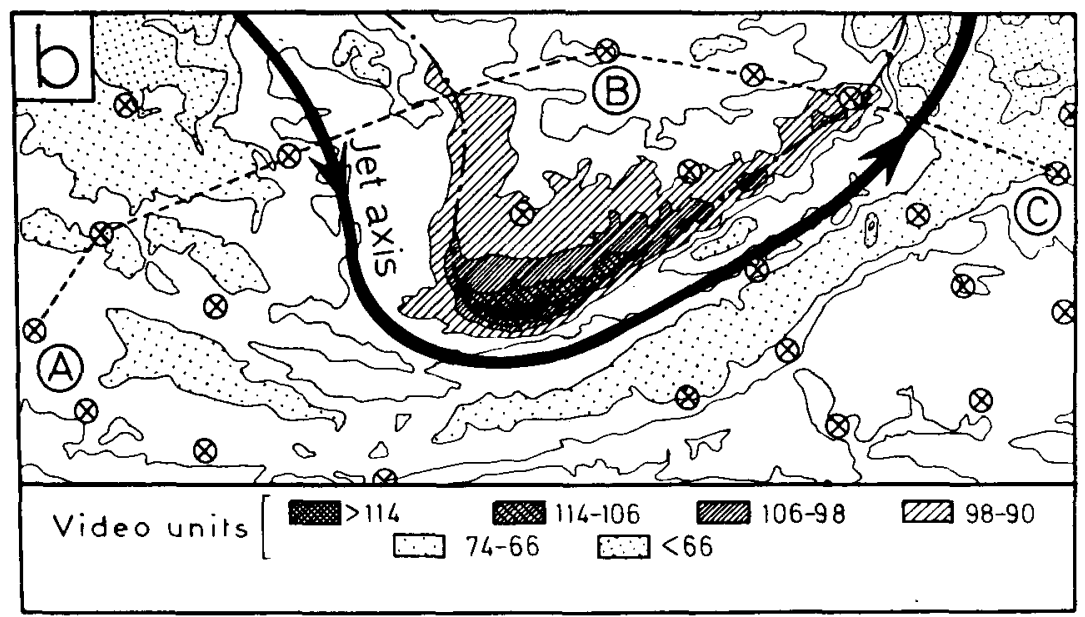

FIG. 1b. Measured METEOSAT WV radiance isocontours (video units) over the same area. 1155 GMT 14 July 1978. METEOSAT projection. Circled crosses are for radiosounding station locations. 


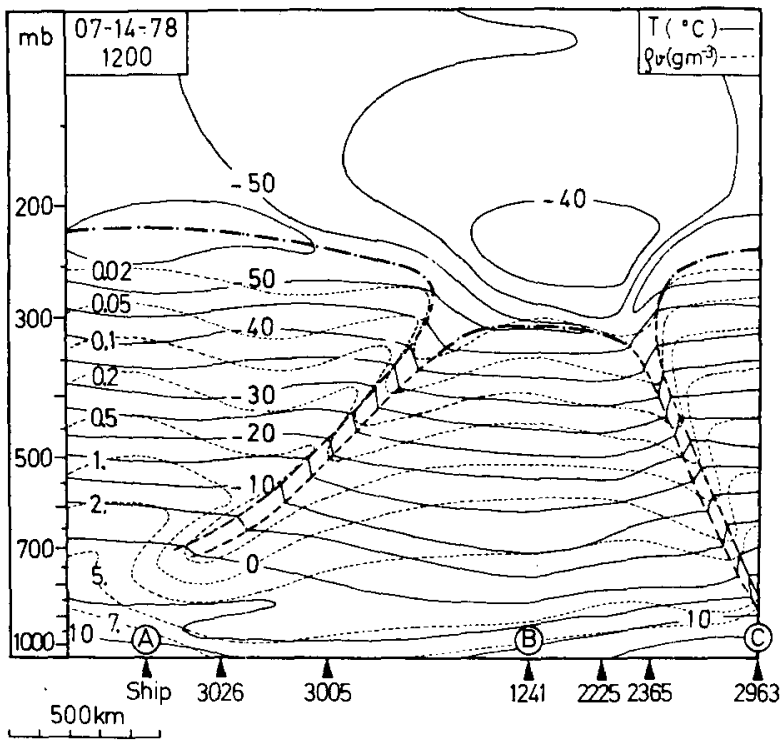

FIG. 2. Vertical cross section along the ABC line of Fig. 1. Full lines, isotherms. Thin dashed lines, water vapor isosteres. Tropopause and frontal layer boundaries are represented by heavy dashed-dotted lines and dashed lines respectively. Radiosounding stations are referred to by their identification number.

whereas maxima should have been expected in the vicinity of the jet streaks on the leading and rear sides of the trough.

The explanation of such a discrepancy has to be looked for in a vertical cross section such as $\mathrm{ABC}$ (Fig. 2) that intersects the southward branch of the jet stream close to and slightly downwind of the jet streak. In this region the air particles that have undergone subsiding motions in the entrance region of the jet streak are likely to begin to rise again, but due to the closeness of the cross section to the jet streak maximum, the global effects of the initial subsiding motions should still be detectable in the vertical cross section. Evidence that strong subsiding motions have taken place on the rear edge of the trough is supplied in Fig. 2 by the shape of the water vapor isosteric contours in the vicinity of the upper frontal zone associated with the jet. Crosssection $A B C$ shown on Fig. 2 is but one element of a subjective isentropic analysis based on a set of 32 vertical cross sections and seven horizontal sections which were drawn and cross checked, using 135 radiosoundings over the European area. Despite a few interpolation errors that may have gone unnoticed, the major features of the thermal and water vapor isosteric fields of cross-section $\mathrm{ABC}$ are fully consistent with the results of other jet stream studies (Palmén and Newton, 1948; Vuorela, 1957; Danielsen, 1968). In particular, it can be seen that the subsiding "dry tongue" follows the frontal zone down to the lower layers and that the underlying cold air also has been subjected to downward motions. Thus, due to the slope of the frontal zone, most of the subsiding (and dry) areas will only be seen by a satellite through the overhanging, relatively moister subtropical air.

Here lies the main difference between the polar jet and the subtropical jet as far as their radiative signatures are concerned. The baroclinic zone associated with the latter is restricted to the upper troposphere; its width is generally larger than that of the polar jet while its slope is smaller. Thus, in the subtropical jet case, as shown schematically in Fig. 3, the satellite can "see down to the bottom" of the subsiding area within the baroclinic zone. The location of the radiance maxima is then correlated with that of the subsidence amplitude maxima.

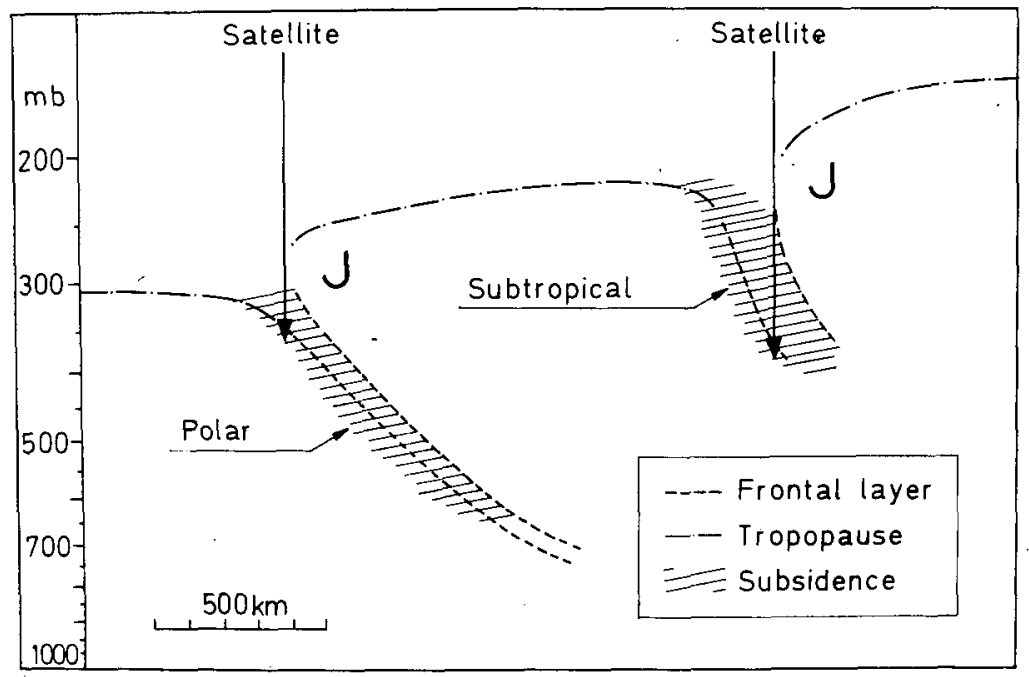

FIG. 3. Schematic representation of polar and subtropical frontal zones. 


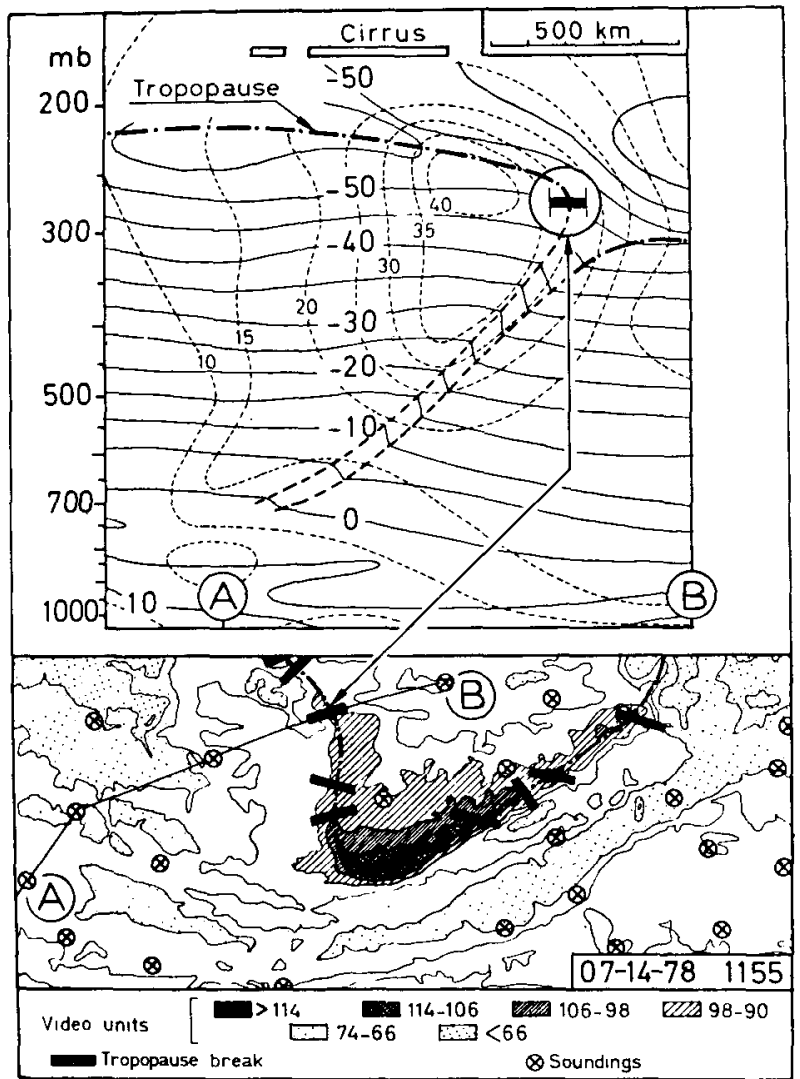

FIG. 4. Top: vertical section AB. Light dashed: isotachs ( $\left.\mathrm{m} \mathrm{s}^{-1}\right)$. Full: isotherms $\left({ }^{\circ} \mathrm{C}\right)$. Heavy dashed dotted: tropopause. Cirrus cover is indicated at top. Bottom: METEOSAT WV radiance field (see Fig. 1). Locations of tropopause breaks along analyzed vertical cross sections are indicated by black rectangles allowing for a $\pm 50 \mathrm{~km}$ error in position.

In the polar jet stream case, a question is still to be answered concerning the interpretation of the dark band in terms of dynamics, since it does not seem to reflect the location of the subsidence amplitude maxima. A first element of answer is found on Fig. 4 where locations of the "tropopause break", deduced from 11 cross-section analyses, are plotted against the radiance field measured on 14 July 1978 . An error of $\pm 50 \mathrm{~km}$ has been allowed for in the interpolated locations. It is clear, at least on this example, that the dark band might be a good tracer of the "tropopause break".

In the following, it will be shown that such is actually the case, in any polar jet stream situation. For this purpose, a necessary first step is to derive an analytic approximation of the radiative transfer equation, appropriate for METEOSAT WV channel; through this approximation the behavior of the contribution function may then be directly assessed.

\section{Analytic approximation of radiative transfer equation}

The spectral response function $\omega(\nu)$ of the METEOSAT WV channel is illustrated in Fig. 5. In the bandwidth of the channel, absorption of other atmospheric gases than water vapor may be neglected in a first approximation. In addition, the amount of water vapor between the surface and the satellite is generally high enough so that the surface emission may be neglected in the total emerging radiance. The radiance transfer equation is then written

$$
N=\int_{z_{0}}^{z_{\infty}} \Psi d z
$$

with

$$
\Psi=\int_{0}^{\infty} B_{\nu}\left(T_{z}\right) \frac{\partial \tau_{\nu}(z, \theta)}{\partial z} \omega(\nu) d \nu
$$

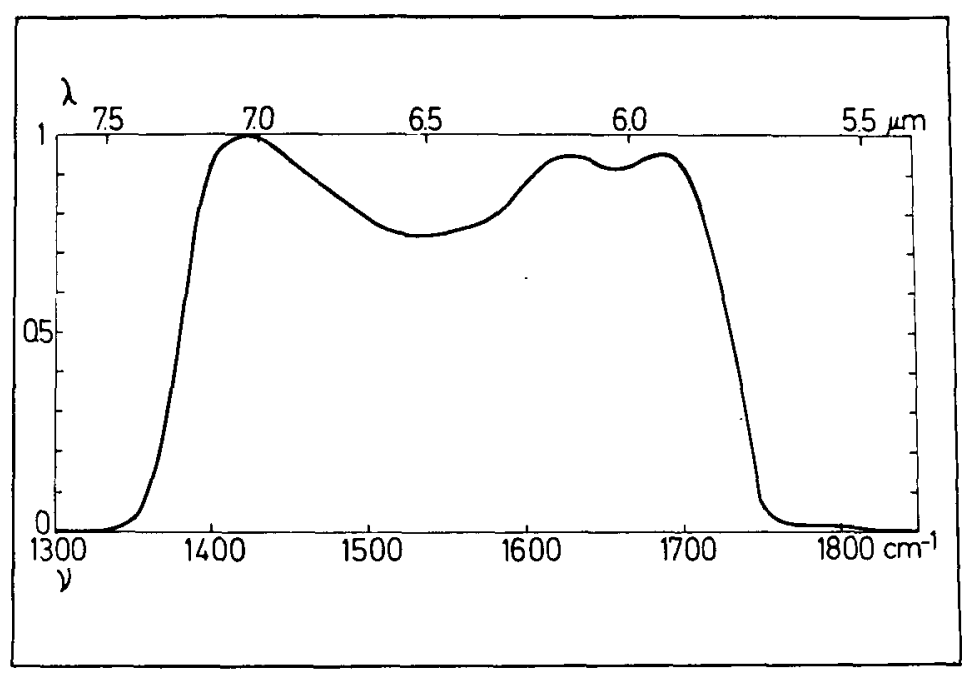

FIG. 5. METEOSAT WV channel normalized spectral response. 
TABLE 1. Water vapor $6.3 \mu \mathrm{m}$ band parameters (after Rodgers and Walshaw, 1966). Subscript $s$ refers to standard temperature and pressure conditions.

\begin{tabular}{|c|c|c|c|c|}
\hline $\begin{array}{l}\text { Interval } \\
\left(\mathrm{cm}^{-1}\right)\end{array}$ & $\begin{array}{c}S_{0} / \delta \\
\left(\mathrm{kg}^{-1} \mathrm{~m}^{2}\right)\end{array}$ & $\pi \alpha / \delta$ & $\begin{array}{c}k_{s} \\
\left(\mathrm{~kg}^{-1} \mathrm{~m}^{2}\right)\end{array}$ & \\
\hline $1200-1350$ & 1.265 & 0.089 & 0.11 & \multirow{7}{*}{$\begin{array}{l}\text { METEOSAT } \\
\text { WV channel }\end{array}$} \\
\hline $1350-1450$ & 13.44 & 0.230 & 3.097 & \\
\hline $1450-1550$ & 63.29 & 0.320 & 20.25 & \\
\hline $1550-1650$ & 32.12 & 0.296 & 9.80 & \\
\hline $1650-1750$ & 43.41 & 0.452 & $19.62]$ & \\
\hline $1750-1850$ & 13.60 & 0.359 & 4.88 & \\
\hline $1850-1950$ & 3.56 & 0.165 & 0.59 & \\
\hline
\end{tabular}

where $N$ is the total emerging radiance, $B_{\nu}(T)$ the Planck function for temperature $T, \tau_{\nu}(z, \theta)$ the transmittance along the line of sight between the satellite and a point at altitude $z ; \theta$ is the zenith angle and $\omega(\nu)$ the spectral response function. Subscripts 0 and $z$ refer to surface and $z$ altitude, respectively; $z_{\infty}$ is for the top of the moist atmosphere. In (1) the effect of earth curvature has been neglected. If a transmittance model is available, the contribution function $\Psi$ may be numerically integrated for any given vertical profile of water vapor and temperature. An alternative way to evaluate $\Psi$ is to rewrite it as follows:

or

$$
\Psi=B_{\nu^{\prime}}\left(T_{z}\right) \int_{0}^{\infty} \frac{\partial \tau_{\nu}(z, \theta)}{\partial z} \omega(\nu) d \nu
$$

$$
\Psi=B_{\nu}\left(T_{z}\right) \frac{\bar{\partial} \tau(z, \theta)}{\partial z} \int_{0}^{\infty} \omega(\nu) d \nu,
$$

where $\nu^{*}$ is an average wavenumber which varies only slightly with altitude (due to variations of the vertical derivative of the transmittance); $\bar{\tau}(z, \theta)$ is a mean transmittance weighted by the spectral response function of the METEOSAT WV channel. As the spectral response curve is close to a top hat profile, it seems reasonable to approximate $\bar{\tau}(z, \theta)$ by an unweighted mean transmittance since our purpose is not to derive quantitative values of the total radiance but rather to study the overall shape of contribution function profiles. The Goody (1952) random line model, which due to the number of absorption lines in the $6.3 \mu \mathrm{m} \mathrm{H}_{2} \mathrm{O}$ band appears as a suitable model, offers an analytical expression $\bar{T}(u)$ for such an unweighted mean transmittance involving a constant pressure optical path $u$ :

with

$$
\bar{T}(u)=\exp \left[-(k u)^{1 / 2}\right],
$$

$$
k=\frac{S_{0} \pi \alpha}{\delta^{2}},
$$

where $k$ is the mass absorption coefficient, $S_{0}$ the mean line strength, $\alpha$ the (Lorentz) line width, $\delta$ the mean line spacing. In $\mathrm{Eq}$. (4) the strong line case assumption has been made. Values of $k$ in the 6.3 $\mu \mathrm{m} \mathrm{H}_{2} \mathrm{O}$ band are given in Table 1 for $100 \mathrm{~cm}^{-1}$ intervals deduced from the results of Rodgers and Walshaw (1966).

These data show that the METEOSAT WV channel encompasses a relatively large spectral range where transmission is rather inhomogeneous, and that a single analytic formulation will only be a rough approximation of the actual transmission over this range. It will be seen in the following, however, that a better approximation is not necessary for our purpose (see Table 1).

Using the Curtis-Godson approximation (Paltridge and Platt, 1976, p. 168), in order to take in account the effects of pressure on the transmission function, and neglecting the atmospheric refraction, (4) is rewritten

$$
\bar{T}(u)=\bar{\tau}(z, \theta)=\exp \left[-\left(K \int_{z}^{z_{\infty}} p_{z^{\prime}} \rho_{z^{\prime}} d z^{\prime}\right)^{1 / 2}\right],
$$

with

$$
K=\frac{k_{s}^{*}}{\cos \theta p_{s}},
$$

where $p$ is the pressure, $\rho$ the water vapor density; the subscript $s$ refers to standard conditions, $k_{s}{ }^{*}$ is a mean mass absorption coefficient over the channel bandwidth. The effects of temperature on the transmission function have not been explicitly considered in the above analytic approximation.

Developing (3) with the aid of (6), we obtain the approximated contribution function $\Psi^{*}$

$$
\begin{aligned}
\Psi^{*}(z)=B_{\nu^{k}}\left(T_{z}\right) \int_{0}^{\infty} \omega(\nu) d \nu & \times\left[1 / 2 p_{z} \rho_{z} K S^{-1 / 2} \exp \left(-S^{1 / 2}\right)\right],
\end{aligned}
$$$$
\text { with }
$$

$$
S=K \int_{z}^{z_{x}} p_{z^{\prime}} \rho_{z^{\prime}} d z^{\prime}
$$

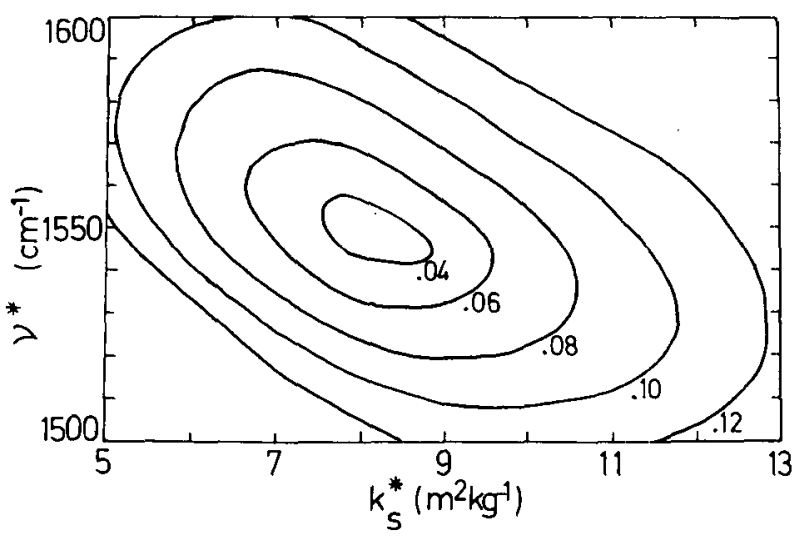

Fig. 6. Bivariate distribution of the standard deviation of $\left(\Psi-\Psi^{*}\right)$. Values are normalized to the average maximum value of the contribution profiles. 
Further use of (7) and (3) requires the determination of the best-fitted values of $\nu^{*}$ and $k_{s}{ }^{*}$ over the whole METEOSAT WV channel. This has been achieved by comparing the values of $\Psi^{*}$, obtained through (7) with different values of $k_{s}^{*}$ and $\nu^{*}$, to those of $\Psi$ resulting from a direct numerical integration of (1) using a generalized parameter transmittance model (LOWTRAN IV, Selby et al., 1978) $)^{1}$ which incorporates temperature corrections on transmittance and absorption by other gases. A set of more than 730 mid-latitude $\left(32-72^{\circ} \mathrm{N}\right)$ radiosoundings scattered over a wide range of meteorological situations has been gathered as a data base, yielding more than 40000 evaluations of $\Psi$, and $\Psi^{*}$. Though in the comparison of $\Psi$ and $\Psi^{*}$ the reliability of the humidity data is of no real importance, all humidity measurements above $250 \mathrm{mb}$ have been rejected. Above $200 \mathrm{mb}$ (or tropopause level when higher) the $\rho$ profile was assumed identical to that given by Selby et al. (1978) for the standard atmosphere.

Fig. 6 gives, as a function of ${k_{s}}^{*}$ and $\nu^{*}$, the twodimensional distribution of the standard deviation of $\left|\Psi^{*}-\Psi\right|$. This quantity has been normalized by dividing by the average $\bar{\Psi}_{\max }$ of the largest values of $\Psi$ in each vertical profile. The radiosounding data base has also been extended by using digitized interpolated fields of experimental vertical cross sections and by artificially letting the zenith angle $\theta$ vary from 0 to $70^{\circ}$. Only a negligible modification of the results was observed. A well-defined minimum of the standard deviation, close to $4 \%$ of the mean peak value $\bar{\Psi}_{\max }$, is reached for $k_{s}{ }^{*} \approx 8 \mathrm{~m}^{2}$ $\mathrm{kg}^{-1}$ and $\nu^{*} \approx 1550 \mathrm{~cm}^{-1}$. These values of $k_{s}{ }^{*}$ and $\nu^{*}$ would then be the best compromise for the fitting of the contribution function but, as it will be seen later, rather than the contribution function, it is the best fitting of an analytic approximation to the weighting function $W$ that is needed. The latter is defined as the integral appearing in (2), and may be written after normalization over the spectral interval

$$
W=\int_{0}^{\infty} \frac{\partial \tau_{\nu}(z, \theta)}{\partial z} \omega(\nu) d \nu / \int_{0}^{\infty} \omega(\nu) d \nu .
$$

Eq. (3) becomes

$$
\Psi^{*}=B_{\nu^{*}} \int_{0}^{\infty} \omega d \nu W^{*},
$$

and according to (7), the analytic approximation $W^{*}$ of the weighting function is expressed by

${ }^{1}$ Selby, J. E. A., F. X. Kneizys, J. H. Chetwynd, Jr., and R. A. McClatchey, 1978: Atmospheric Transmittance/Radiance: Computer code LOWTRAN IV. A.F.G.L.-TR-78-0053 Air Force Geophysics Laboratory, Hanscom AFB, Mass., 100 pp. [NTIS N79-13624].

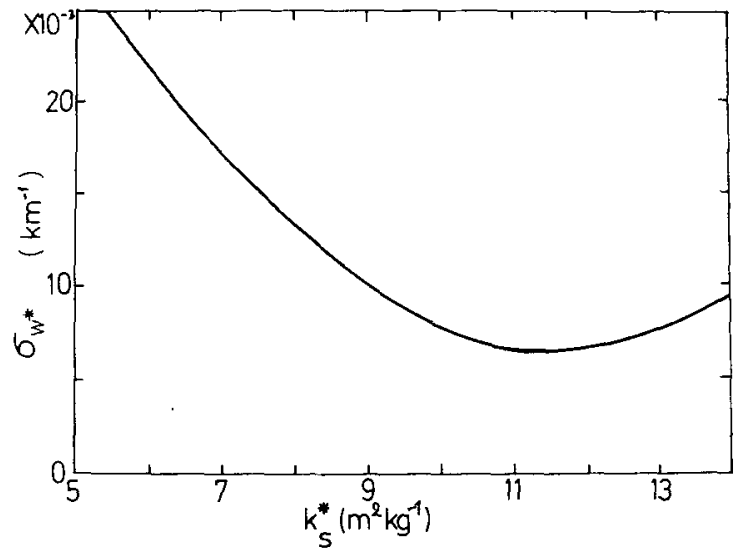

FIG. 7. Distribution of the standard deviation of $\left(W-W^{*}\right)$ according to the mean mass absorption coefficient $K$.

$$
W^{*}=1 / 2 p \rho K S^{-1 / 2} \exp \left(-S^{1 / 2}\right) .
$$

Fig. 7 gives the distribution of the standard deviation of $W^{*}$ computed through (10) with different values of $k_{s}{ }^{*}$, as compared to $W$ computed through (8) with the use of the LOWTRAN IV transmittance model. The data base for these computations is the same as above for $\Psi$. A minimum standard deviation is reached for $k_{s}^{*} \approx 11 \mathrm{~m}^{2} \mathrm{~kg}^{-1}$, a value which is slightly higher than the optimal value found when fitting $\Psi^{*}$. This discrepancy can be expected as, for a mean value of $k_{s}{ }^{*}$ around 10 , the analytic approximation fails to simulate satisfactorily the radiative processes in the 1350 $1450 \mathrm{~cm}^{-1}$ band, where the atmospheric absorption is quite low $\left(k_{s}=3.09 \mathrm{~m}^{2} \mathrm{~kg}^{-1}\right.$, see Table 1). This particular spectral band actually produces a spreading toward the lower layers of the width of the contribution function profile (CFP). Taking the best fit found for the weighting function $\left(k_{s}{ }^{*}=11 \mathrm{~m}^{2}\right.$ $\mathrm{kg}^{-1}$ ) will result into an underestimation of the contribution function in the lower layers. This effect may be reduced by a compromise which consists in decreasing somewhat the value of $k_{s}{ }^{*}$ thus departing slightly from the best fit of the weighting function. As such a compromise, the following values of $k_{s}{ }^{*}$ and $\nu^{*}$ have been selected:

$$
\left.\begin{array}{rl}
k_{s}^{*} & =10 \mathrm{~m}^{2} \mathrm{~kg}^{-1} \\
\nu^{*} & =1540 \mathrm{~cm}^{-1}
\end{array}\right\}
$$

for which the normalized standard deviations of $\left|W^{*}-W\right|$ and $\left|\Psi^{*}-\Psi\right|$ are close to 4 and $7 \%$, respectively.

Fig. 8a gives an example of vertical profiles obtained with the above values. As expected, the analytic Weighting Function Profile (WFP) appears visually as shifted downward relatively to its bestfit position while the underestimation of the contribution function, though it does not disappear, is reduced in the lower layers. 


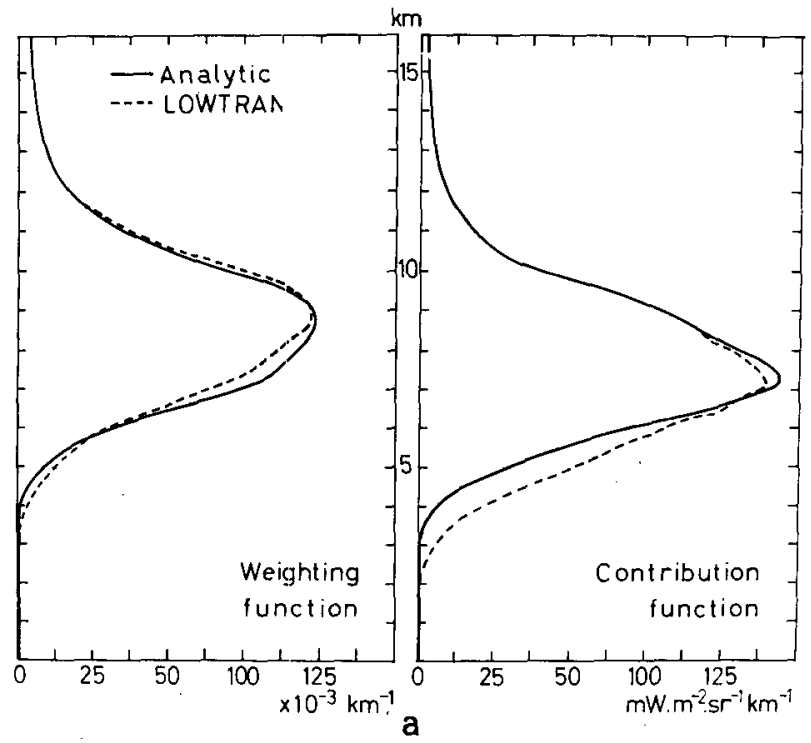

FIG. 8a. Example of WFP and CFP for an actual air mass sounding. Results of the analytic approximation are compared to those of the LOWTRAN IV transmittance model.

As a further check the results of the analytic approximation, concerning the barycenter altitude of the vertical profiles and their second-order moments about this altitude, were compared to those obtained with the LOWTRAN IV transmittance model. Table 2 summarizes the results of the comparison; all the data have been normalized by dividing by the square root of the secondorder moments about the barycenter altitude, which give a measure of the vertical width of the profiles. About $20 \%$ of the vertical soundings

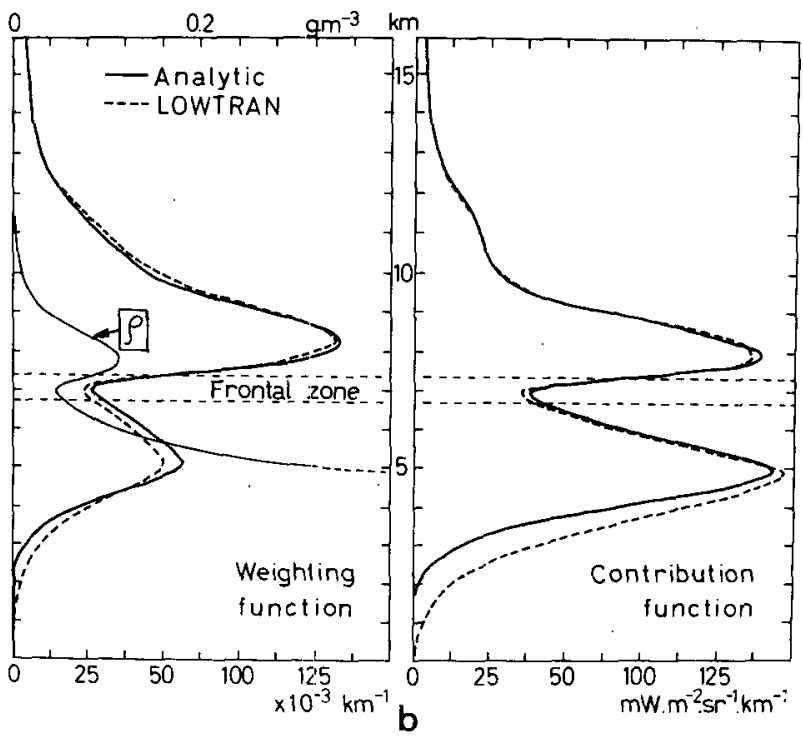

FIG. 8b. As in Fig. 8a but for a sounding crossing an upper frontal layer. The vertical $\rho$ profile is also drawn. involved in this analysis were intersecting frontal layers. Figure $8 \mathrm{~b}$ shows typical vertical profiles of $W, W^{*}, \Psi$ and $\Psi^{*}$ in such cases. As noted above the contribution function is again slightly underestimated in the lower layers by the analytic approximation. However, it can be seen that despite this shortcoming the analytic approximation gives rather good results even in such complex cases. This, added to the results of Table 2 , validates the use of the analytic approximation.

In the next sections, a closer analysis of the behavior of the CFP and WFP, using their analytic approximations (9) and (10), will be done separately for the non-frontal and frontal situations.

\section{Air mass case \\ a. A $\rho$-profile model}

Discussing directly the behavior of the CFP and WFP from (9) and (10) requires a suitable model of the vertical profile of the product $p \rho$. Within the limit of accuracy needed for our purpose, an exponential profile can be taken for the pressure

$$
p=p_{0} \exp \left(-z / H_{p}\right),
$$

where the pressure scale height $H_{p}$ is close to $7600 \mathrm{~m}$.

As for the $\rho$ profile, the following model has been tested statistically on the radiosounding data base described earlier:

$$
\rho=\rho_{0} \exp \left(-z / H_{\rho}\right) .
$$

The contribution to the total radiance of the layers located below $600 \mathrm{mb}$ is always negligible and the goodness of the fit is only required above this level. The humidity soundings that did not reach the 400 mb level ( $10 \%$ of the total number) were eliminated from the data base. All measurements above $250 \mathrm{mb}$ were discarded and the exponential law of (13) was considered to be representative of the sharp decrease of the water vapor density with height in the upper troposphere and lower stratosphere, where reliable measurements are very scarce. For each sounding the data were interpolated every $200 \mathrm{~m}$

TABLE 2. Comparison of the barycenter altitudes $\bar{z}^{*}$ and second-order moments $m_{2}{ }^{*}$ of the approximated profiles with those computed with the LOWTRAN IV transmittance model $\left(\bar{z}, m_{2}\right)$. Data are normalized to the square root of the second-

\begin{tabular}{|c|c|c|c|}
\hline & . & $\begin{array}{l}\text { Contribution } \\
\text { function }\end{array}$ & $\begin{array}{l}\text { Weighting } \\
\text { function }\end{array}$ \\
\hline $\bar{z}-\bar{z}^{*}$ & $\begin{array}{l}\mid \text { mean } \mid \\
\text { standard deviation }\end{array}$ & $\begin{array}{l}1.610^{-3} \\
4.110^{-2}\end{array}$ & $\begin{array}{ll}3.5 & 10^{-2} \\
2.1 & 10^{-2}\end{array}$ \\
\hline$\sqrt{m_{2}}-\sqrt{m_{2}{ }^{*}}$ & $\begin{array}{l}\mid \text { mean } \mid \\
\text { standard deviation }\end{array}$ & $\begin{array}{l}6.110^{-2} \\
1.610^{-2}\end{array}$ & $\begin{array}{l}2.110^{-2} \\
1.310^{-2}\end{array}$ \\
\hline
\end{tabular}
order moments. 
in altitude according to the WMO standards and fitted to (13) through a least-squares procedure yielding the values of $\rho_{0}, H_{\rho}$ and the standard deviation $s$ of $\ln (\rho)$. About $20 \%$ of the data base consisted of soundings that crossed frontal layers either polar or subtropical and should have been removed from the data. No objective criteria, however, have been found for this removal but a direct examination of the soundings (and corresponding synoptic contexts) that formed the wings of the distribution spectra made possible a partial elimination of the frontal soundings. As a matter of fact every sounding that led to values of $s$ and $H_{\rho}$ such that $s>0.4$, $H_{\rho}>2700 \mathrm{~m}$ or $H_{\rho}<900 \mathrm{~m}$ proved to be a frontal sounding. With these criteria one third of the frontal soundings could be eliminated. The contamination of the results due to the remaining ones was judged sufficiently small so as to make useless a further refinement of the rejection criteria.

Fig. 9 presents an histogram of the standard deviation $s$. Despite the presence of frontal soundings mostly in the wings of the histogram - the results indicate, using the model given by (13), that $\sim 80 \%$ of experimental water vapor density profiles are retrieved with an accuracy better than 20\%; therefore, this model may be retained as a good first approximation of the vertical $\rho$ profiles within air masses.

While the distribution of the parameter $\rho_{0}$ was found to be widely scattered, the results of Table 3 show that the scale height $H_{\rho}$ is quasi-constant, even

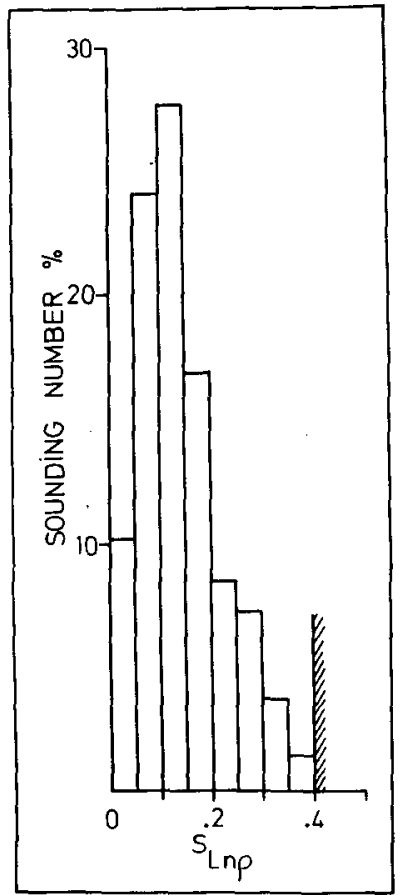

FIG. 9. Histogram of the standard deviation $s$ of $\ln \rho$.
TABLE 3. Value and standard deviation of retrieved exponential $\rho$-profile scale heights.

\begin{tabular}{lcc}
\hline Air mass & $\begin{array}{c}\text { Average scale } \\
\text { height }(\mathrm{m})\end{array}$ & $\begin{array}{c}\text { Average standard } \\
\text { deviation }(\mathrm{m})\end{array}$ \\
\hline Tropical and & & \\
subtropical & 1570 & 265 \\
Polar & 1360 & 160 \\
Total & 1560 & 270 \\
\hline
\end{tabular}

if it is possible to discriminate the scale heights of air masses according to their origin. The narrow distribution of the water vapor density scale height is an important argument of the following discussion. In this discussion, $H_{\rho}$ will be considered as constant and equal to $1560 \mathrm{~m}$; the product $p \rho$ then reads

$$
p \rho=p_{0} \rho_{0} \exp (-z / H)
$$

with

$$
H=\left[\frac{1}{H_{p}}+\frac{1}{H_{\rho}}\right]^{-1} \approx 1300 \mathrm{~m}
$$

\section{b. Altitude of the weighting function peak}

The vertical logarithmic derivative of the weighting function $W^{*}$ is derived from (10) giving

$$
\frac{1}{W^{*}} \frac{d W^{*}}{d z}=\frac{K p \rho}{2 S}\left(S^{1 / 2}+1\right)+\frac{1}{p \rho} \frac{d(p \rho)}{d z} .
$$

Using (14) and equating (15) to zero one obtains the altitude $z_{W}$ of the peak of the weighting function

$$
z_{W}=H \ln \left(K H p_{0} \rho_{0}\right) .
$$

As the distribution of the parameter $\rho_{0}$ has been found rather scattered little can be said $a$ priori on $z_{W}$. However, using once more (14) one may write

$$
(p \rho)_{\boldsymbol{x}_{W}}=\frac{1}{K H}=\frac{p_{s} \cos \theta}{k_{s}^{*} H},
$$

where $p_{s}, k_{s}^{*}$ and $H$ are constant. Eq. (17) is independent of $\rho_{0}$ and indicates that as inasmuch as zenith angle $\theta$ can be considered as constant, that is, when the analyzed area is of limited extent across $\theta$ isocontours, then the peak of the weighting function is located on a surface where the product $p \rho$ is constant. Since the pressure scale height $H_{p}$ is much larger than the water vapor density scale height and since, within air masses, the altitude variations of the water vapor isosteric surfaces in the middle and upper atmosphere are but a small fraction of $H_{p}$, equating $p \rho$ to a constant is nearly equivalent to equating $\rho$ to a constant.

Thus, provided the zenith angle does not vary too much, the peak of the weighting function in a "homogeneous" air mass is located on a water vapor isosteric surface. 


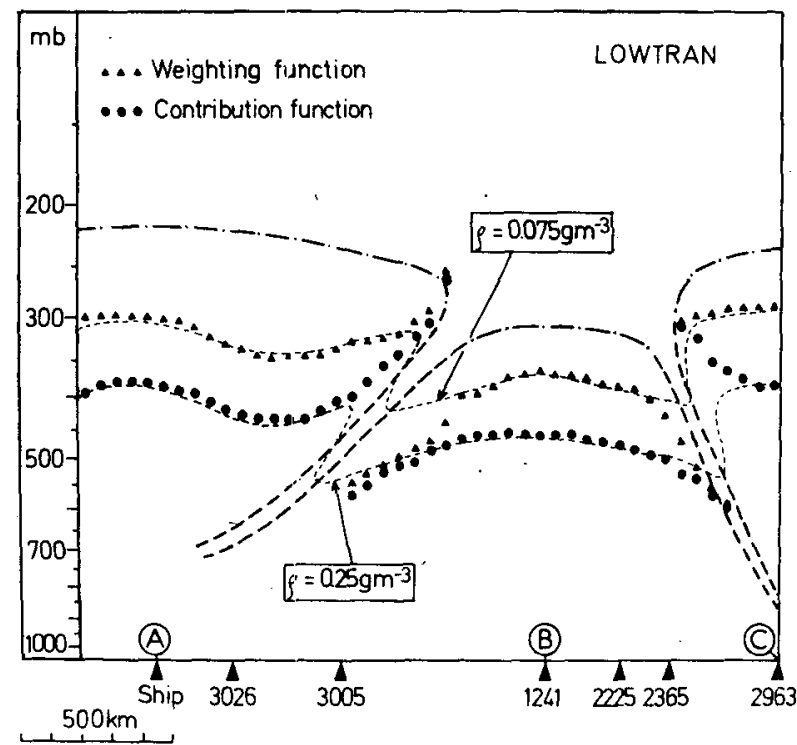

FIG, 10. Comparison of the altitudes of the WFP and CFP peaks determined with LOWTRAN IV model, and those of two water vapor isosteric surfaces $\left(\rho=0.075 \mathrm{~g} \mathrm{~m}^{-3}\right.$ and $\rho=0.25$ $\mathrm{g} \mathrm{m}^{-3}$ ) along vertical cross-section $\mathrm{ABC}$.

Fig. 10 illustrates an experimental verification of this theoretical. result. It has been deduced from Fig. 2 after digitizing the isocontours and computing the weighting and contribution profiles every $50 \mathrm{~km}$ along the vertical section, using the LOWTRAN IV transmittance model. The location of the peak on each profile was defined as the location of the barycenter of points where the value of the function exceeded $90 \%$ of the maximum value.

In the vertical section $A B C$ of Fig. 10, the zenith angle $\theta$ varies from 64 to $72^{\circ}$, and it can be verified that away from the frontal zones the altitude of the peak of the weighting function is very close to that of an isosteric contour $\left(\rho=0.075 \mathrm{~g} \mathrm{~m}^{-3}\right)$. Note that it is the same isosteric contour in the warm air and the cold air, which illustrates the fact that the water vapor density scale height $H_{\rho}$ is hardly different from one air mass to the other as established by the statistical study of water vapor profiles reported in Section 3a.

It can be seen on Fig. 10 that similar conclusions seem to apply also to the contribution function profile. The altitude $z_{C}$ of the peak of this profile is the solution of the equation

$$
\frac{1}{\Psi^{*}} \frac{d \Psi^{*}}{d z}=\frac{1}{W^{*}} \frac{d W^{*}}{d z}+\frac{1}{B_{\nu^{*}}} \frac{d B_{\nu^{*}}}{d T} \frac{d T}{d z}=0 .
$$

If we take as $z$ origin the altitude $z_{W}$ of the WFP peak, (10) is rewritten with the use of (14)

$$
W^{*}=\frac{1}{2 H} \exp \left[-\exp \left(-\frac{Z}{2 H}\right)-\frac{Z}{2 H}\right] \text {, }
$$

with

$$
Z=z-z_{W} .
$$

From the Planck function we have also

$$
\frac{1}{B_{\nu^{*}}} \frac{d B_{\nu^{*}}}{d T} \frac{d T}{d z} \approx \frac{\beta_{\nu^{*}} \gamma}{T^{2}}
$$

where $\beta_{\nu^{*}}$, expressed in Kelvin, is a constant $\left(=1.438 \nu^{*}\right)$ and $\gamma\left(\mathrm{K} \mathrm{m}^{-1}\right)$ is the vertical temperature gradient which we assume constant within the layer separating the WFP and CFP peaks.

Replacing (19) and (20) in (18), the altitude of the CFP peak relative to that of the WFP is finally given by

$$
Z_{C}=-2 H \ln \left(1-2 H \frac{\beta_{v^{*}} \gamma}{T_{C}^{2}}\right),
$$

where $T_{C}$ is the temperature at the CFP peak level.

As the average temperature gradient $\gamma$ between the WFP and CFP peaks is normally negative, the term within brackets of (21) is larger than 1 . Its value for the standard atmosphere given by Selby et al. (1968) is 1.6, which yields, according (21), $Z_{C}$ $=-1230 \mathrm{~m}$, a value which agrees within $10 \%$ with that computed with the LOWTRAN IV transmittance model.

The scale height $H$ being quasi-constant, $Z_{C}$ will be considered as constant provided fluctuations, within an air mass, of the logarithmic factor of (21) can be neglected. However, when one considers realistic variations of $\gamma$ and $T_{c}$ in an air mass $( \pm 1.5$ $\times 10^{-3} \mathrm{~K} \mathrm{~m}^{-1}$ and $\pm 10 \mathrm{~K}$, respectively) one obtains a value of $25 \%$ for the relative fluctuation of the logarithmic term, which is too high to be neglected in a first order approximation but nevertheless small enough to conclude that the CFP peak within an air mass lies very close to a water vapor isosteric surface.

\section{c. Significance of the radiance field}

Further interesting consequences concerning the WFP may be derived from (19) as follows:

1) The WFP peak is located at a level where the optical depth, $K \int_{z}^{z_{\infty}} p \rho d z$, is equal to unity.

2) As long as $H$ is constant the WFP is invariant, the effect of any variation of $k_{s}, \rho_{0}, p_{0}$ is a mere bulk translation along the vertical.

This latter remark suggests that choosing the WFP peak level as the altitude origin should be of some advantage for the interpretation of the radiative transfer. Eq. (1) is then rewritten with the aid of (9) and (19)

$$
\begin{aligned}
N=\frac{\delta}{2 H} \int_{-z_{W}}^{z_{\infty}} B_{\nu^{\prime}}\left(T_{Z}\right) & \\
& \times \exp \left[-\exp \left(-\frac{Z}{2 H}\right)-\frac{Z}{2 H}\right] d Z,
\end{aligned}
$$


where $\delta=\int_{0}^{\infty} \omega(\nu) d_{\nu}$. In the simple case of a thermally stratified air mass $(\gamma=$ const., $d T / d x=0)$, the temperature $T_{Z}$ at the altitude $Z$ is given by

$$
T_{Z}=T_{W}+\gamma Z,
$$

and $z_{W}$ is uniquely determined by the temperature $T_{W}$ at the WFP peak level. The total emerging radiance $N$ given by (22) appears as a function of $T_{W}$ alone

$$
N=f\left(T_{W}\right) .
$$

The radiance field would thus, in this simple case, reflect the field of temperature $T_{W}$ at WFP peak level, that is the temperature field on a water vapor isosteric surface.

Furthermore, it will now be shown that this also is true even when rather large variations of $\gamma$ are present from one point to another across the air mass. Let us assume that each temperature sounding can be approximated by defining a constant lapse rate $\gamma$ in the middle and upper troposphere and that above the tropopause altitude $Z_{\text {tropo }}$ the temperature $T_{\text {strato }}$ is constant with height; the temperature $T_{Z}$ at altitude $Z$ relatively to WFP peak is given by

$$
\left.\begin{array}{l}
T_{Z}=T_{W}+\gamma Z, \quad Z<Z_{\text {tropo }} \\
T_{\text {strato }}=T_{W}+\gamma Z_{\text {tropo }}=c^{\text {ste }}
\end{array}\right\} .
$$

The Planck function may be approximated by

where

$$
\begin{aligned}
B_{\nu}\left(T_{z}\right) & \simeq \alpha_{\nu} \exp \left(-\frac{\beta_{\nu}}{T_{z}}\right) \\
& \simeq \alpha_{\nu} \exp \left[-\frac{\beta_{\nu}}{T_{W}}\left(1-\frac{\gamma Z}{T_{W}}\right)\right],
\end{aligned}
$$

$$
\alpha_{\nu}=1.191 \times 10^{-12} \nu^{3}\left(\mathrm{~W} \mathrm{~cm}^{-2} \mathrm{~cm} \mathrm{sr}^{-1}\right) .
$$

Using (22), (23) and (24) and neglecting terms of order higher than two (or alternatively assuming a null value of the weighting function at the surface), the total radiance is given by

$$
N=\alpha_{\nu}: \delta \exp \left(-\frac{\beta_{\nu^{*}}}{T_{W}}\right) \cdot \theta,
$$

where

$$
\begin{aligned}
\Theta & =\left\{\Gamma(\epsilon) Q\left(2 \mu_{t} / 2 \epsilon\right)+\mu_{t}^{\epsilon-1}\left[1-\exp \left(-\mu_{t}\right)\right]\right\}, \\
\epsilon & =1-\frac{2 H \beta_{v^{*}} \gamma}{T_{W}^{2}}, \\
\mu_{t} & =\left[K H(p \rho)_{\text {tropopause }}\right]^{1 / 2} .
\end{aligned}
$$

$\Gamma$ is the gamma function and $Q$ is the chi-square probability function. Taking for example the following wide ranges of variation of $\gamma, T_{W}$ and $\mu_{t}$, which are far in excess of actual variations in individual air masses

$$
\begin{aligned}
-8.5 \times 10^{-3} \mathrm{~K} \mathrm{~m}^{-1} & <\gamma<-5 \times 10^{-3} \mathrm{~K} \mathrm{~m}^{-1}, \\
225 \mathrm{~K} & <T_{W}<255 \mathrm{~K}, \\
0.05 & <\mu_{t}<0.65
\end{aligned}
$$

the factor $\theta$ in (25) varies only from 0.89 to 1.12 . It may be considered as constant in a first-order approximation, since at the same time the Planck exponential term appearing in (25) is multiplied by a factor exceeding 3 .

If we now define the equivalent blackbody temperature $T_{B B}$ by the following equation

$$
N=\int_{0}^{\infty} B_{\nu}\left(T_{B B}\right) \omega(\nu) d \nu,
$$

where $N$ is the actual measured radiance, we can write

$$
N=B_{\nu 1}\left(T_{B B}\right) \int_{0}^{\infty} \omega(\nu) d \nu .
$$

In the above expression, $\nu_{1}$ is a mean wavenumber. As the METEOSAT WV channel response curve assimilates to a top-hat profile, $\nu_{1}$ is very close to the wavenumber $\nu^{*}$ defined earlier and will be taken equal to it. Then equating the right-hand sides of (25) and (27), and rearranging the terms, one comes to

$$
T_{B B}=T_{W}\left[1 /\left(1-\frac{T_{W}}{\beta_{v^{2}}} \ln \theta\right)\right] .
$$

The magnitude of the last term within parenthesis being $10^{-2}$, the difference between the equivalent blackbody temperature $T_{B B}$ and the temperature at the WFP peak level is approximated by

$$
T_{B B}-T_{W}=\left(T^{2}{ }_{W} / \mathrm{B}_{\nu^{\prime}}\right) \ln \theta .
$$

For the variation ranges given by (26), which are far exceeding actual conditions in individual air masses, this difference evaluated from (28) does not exceed $\pm 3 \mathrm{~K}$. Evaluating the right-hand side term of (28) from the radiosounding data base described in Section 2 which consists of a wide variety of air mass soundings (tropical, subtropical, polar, frontal), gives an average value of $-0.4 \mathrm{~K}$ with a standard deviation equal to $1.5 \mathrm{~K}$. As the corresponding variation range of $T_{W}$ is $\sim 30 \mathrm{~K}$, the difference between the equivalent blackbody temperature and the temperature at WFP peak level may be considered as negligible in a first-order approximation. Thus, the equivalent blackbody temperature field is representative of the air temperature field at the altitude of the WFP peak and, as long as the range of variation of the zenith angle $\theta$ remains small, it is also representative of the air temperature field on a water vapor isosteric surface. This may be verified on Fig. 11 where the measured METEOSAT WV radiance is plotted 


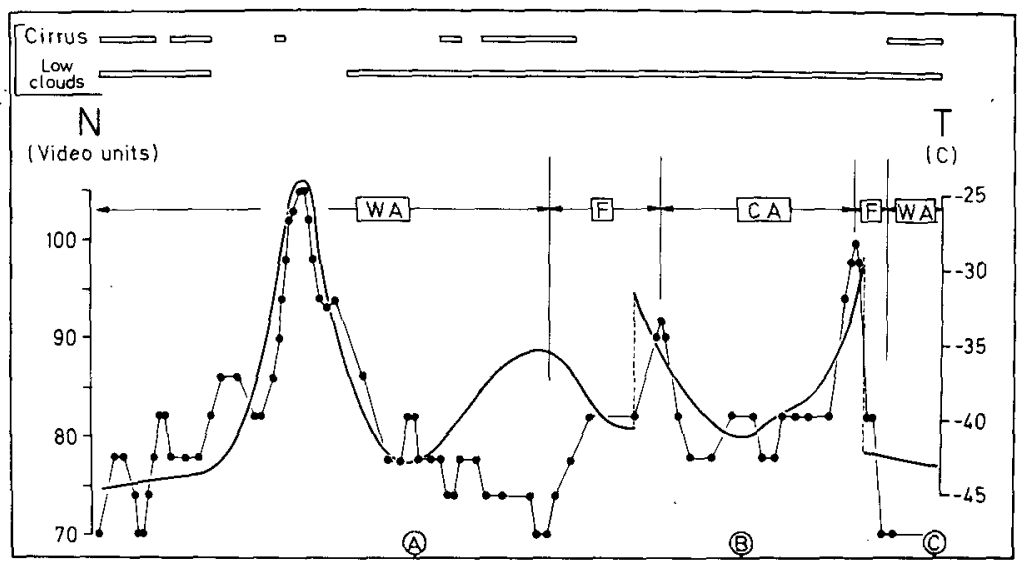

Fig. 11. METEOSAT measured radiance $N$ (dots) and temperature on the water vapor isosteric surface $\rho=0.075 \mathrm{~g} \mathrm{~m}^{-3}$ (heavy line) along the extended vertical cross section $A B C$. WA is for warm air, $C A$ for cold air, and $F$ for frontal layer above $500 \mathrm{mb}$ (see Fig. 2). High and low cloud cover is indicated at the top of the figure.

against the temperature of the isosteric surface $\rho=0.075 \mathrm{~g} \mathrm{~m}^{-3}$ along the vertical section $\mathrm{ABC}$ (see-Fig. 2) that has been purposely extended southwestward beyond point $A$, in order to encompass a particularly dry and narrow band detected aloft by the radiosounding network and viewed as a well-defined dark band on the METEOSAT WV imagery. It can be seen that, away from the frontal zones, and discarding small-scale features that are smoothed by the isosteric temperature interpolation, the measured total radiance follows the variations of the temperature along the water vapor isosteric surface. This agreement, as it would be expected, does not hold in areas where dense cirrus clouds are present. When drawing Fig. 11, the variations of the zenith angle $\theta$ (from 52 to $72^{\circ} \mathrm{N}$ ) have been neglected and a unique isosteric surface was considered over the whole cross section. The agreement of the two curves of Fig. 11 suggests that, as far as first-order approximation is sought, the hypothesis of a constant zenith angle may be omitted in the theoretical deductions made above, for many practical cases.

Before discussing in detail in the next section the radiative transfer across a frontal layer, note that on Fig. 11, apart from a slight horizontal shifting of their maxima, the radiance and isosteric temperature fields still exhibit the same global features, even very near the frontal zones.

\section{Crossing a frontal layer}

Due to subsiding motions that occur within it, at least in the upper troposphere, a polar frontal zone appears as a shallow layer of relatively low water content where sharp variations of $1 / p \rho d(p \rho) / d z$ are to be expected along the vertical.
Across a frontal layer the first term of the righthand side of (15) is only slowly varying as compared to $1 / p \rho d(p \rho) / d z$ which reaches a maximum in the upper part of the layer. These variations yield a depression of the $W^{*}$ profile in the vicinity of the frontal layer.

In most cases the maximum value (often positive) reached by $1 / p \rho d(p \rho) / d z$ in such conditions is sufficient so that the right hand side of (15) becomes positive. Thus, a WFP peak is to be expected in the warm air above the frontal layer, even if the latter is encountered above the theoretical altitude of the WFP peak that would be computed by using (16) with the warm air characteristics. This conclusion also applies to the CFP, since the second term on the right-hand side of (18), following the variations of the vertical temperature gradient, is known to increase sharply and sometimes to become positive within frontal layers and since the first term of (18) takes high positive values in the upper part of the frontal layer and just above it (see above). As shown on Fig. 8b, secondary.WFP and CFP peaks may be found in the cold air below the frontal layers. However, these peaks are likely to occur only when the intersection of the frontal layer and the sight path lies in the upper troposphere $(p<500 \mathrm{mb})$. As a matter of fact, it can be shown, using (14), that in both cold and warm air masses, the first right-hand side term of (15) increases exponentially as altitude decreases, and thus reaches in the lower troposphere high positive values that may no longer be balanced by the second right-hand side term, the absolute value of which remains relatively small within the lower part of and below the frontal layer due to the subsiding motions that affect also the whole underlying cold air mass.

Eq. (18) indicates that wherever a WFP peak does 
exist below the frontal layer, then a CFP peak has also to occur at a lower altitude, since in the cold air the second right-hand side term of (18) is negative.

The above conclusions are in agreement with experimental data, a sample of which is illustrated by Fig. 10, where the altitudes of the WFP and CFP peaks have been computed using the LOWTRAN IV transmittance model (Selby et al., 1978).

\section{Tropopause break and radiance maximum}

Fig. 12 duplicates Fig. 2 with the altitudes of the WFP and CFP peaks added. As it has been said above, the main features of the thermodynamic fields depicted in this figure are in agreement with numerous other observations. Among these features two are of peculiar importance for our purpose:

1) In the cold air, the isotherms are sloping down toward the cold air mass center.

2) Due to subsidence within the frontal layer and the cold air mass, the water vapor isosteres in the cold air are sloping down toward the warm air.

The boundary between zones $b$ and $c$ on Fig. 12 goes through the tropopause break which is defined in a vertical cross section as the furthest point reached poleward by the overhanging warm and moist subtropical air.

On the right side of this boundary, due to the reversed slopes of the isotherms and water vapor isosteres within the cold air, the WV channel radiance that is related to the temperature on a water vapor isostere will decrease as the distance from the boundary increases in zone $c$.

On the left side of the boundary in zone $b$, the sharp increase of the absorption by the overhanging wet air of the radiation rising up from below and the growing contribution of layers with lower temperature located close to the secondary CFP peaks above the frontal layer, will result also in a decrease of the radiance as the distance from the tropopause break boundary increases in zone $b$.

The tropopause break appears thus as being directly associated with a local radiance maximum in the WV channel.

In zone a, an air mass regime is reached and the radiance field will reflect the temperature along an isosteric surface, as long as the zenith angle does not vary significantly.

These inferred variations of the WV channel radiance field are indeed those actually observed (see Figs. 4 and 11), in areas with no high-level clouds.

\section{Cloud-cover effect}

The computations of the radiative transfer that have been so far presented were based on a no cloud assumption, and the experimental data of Fig. 11

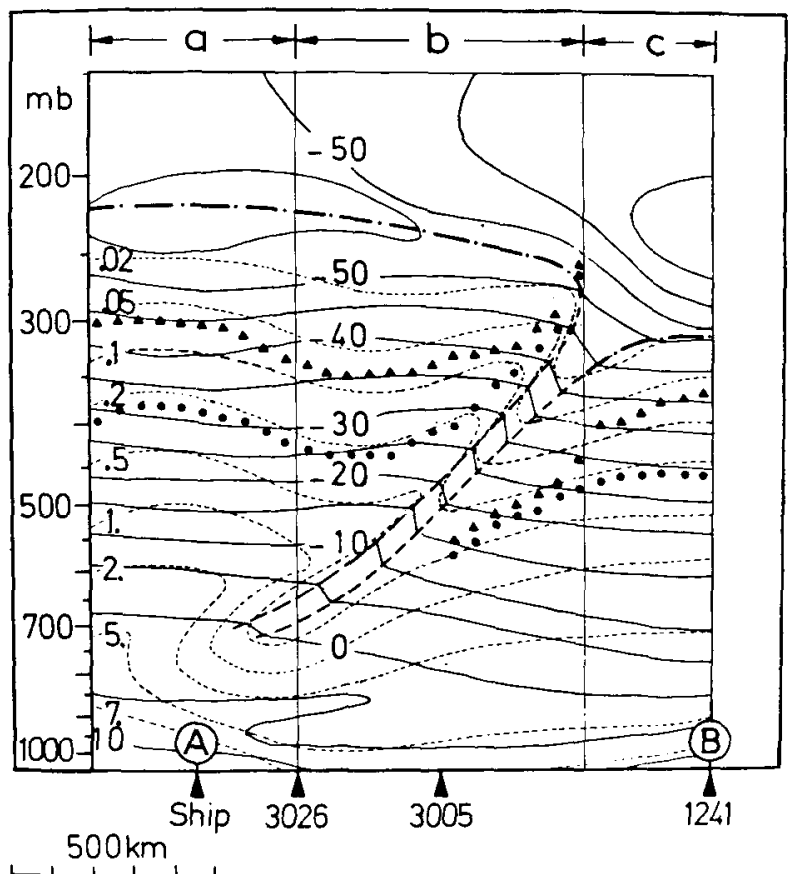

FIG. 12. Vertical cross-section AB. See legend of Fig. 2. Black circles and triangles are for the altitudes of the CFP and WFP peaks, respectively, as determined by LOWTRAN IV model (as Fig. 10). For zones $a, b$ and $c$ see text.

exhibit clearly the contaminating effect of the presence of high-level clouds. However, as the main purpose of this paper is to show that the tropopause break is associated with a radiance maximum in the WV channel, it is obvious that the cloud cover, if any, does not invalidate our conclusions but on the contrary strengthens them. In the entrance region the subsiding motions which are prevailing on the cyclonic side of the jet stream, at tropopause level and within the cold-air mass, prevent the formation of high-altitude clouds and limits the low cloud tops to altitudes where their effects on the total radiance in the WV channel are small. On the other hand, high-altitude clouds (cirrus clouds and convective clouds) may be expected on the anticyclonic side of the jet stream, thus actually increasing the contrast of the tropopause break radiance maximum as exemplified by Figs. 1 and 11 . In the delta region upward motions are prevailing and cold-topped clouds are often found in the vicinity of the jet on both sides of its axis, but in most cases they appear only within the warm air. Since the isothermal surfaces within the cold air are still sloping down toward the cold air mass center even in the delta region as exemplified by Fig. 12, a radiance maximum is again to be found at the tropopause break with an enhanced contrast due to the neighboring cold-topped clouds. Such a case can be seen in Fig. 1 where to the south-southeast of point $B$, a small cirrus band is located between 
the maximum radiance line (tropopause break) and the jet axis.

\section{Conclusions}

It has been shown that the WV radiance signature of the polar jet stream differs significantly from that of the subtropical jet stream. The band of maximum radiance associated with the former delineates the tropopause break, rather than the location of the maximum amplitudes of the subsiding motions as it is the case for a subtropical jet stream. This difference has been shown to be a consequence of the relatively large tilting angle and small thickness of the polar frontal surface. Such a result appears of importance concerning the current attempts to retrieve the vertical velocities in the upper troposphere through the analysis of consecutive WV images from geostationary or low altitude satellites. In this respect, the polar jet stream area, where a large amount of the stratosphere-troposphere exchange is taking place, deserves a special treatment that is far from being as straightforward as in other areas, including the subtropical jet stream. A by-product of the study is the relation found between the WV radiance field and the temperature on a water vapor isosteric surface which, associated with an isentropic hypothesis, may constitute the base of a method for retrieving the mean vertical velocities in the upper troposphere away from the polar frontal zones.

Finally, METEOSAT, though a geostationary satellite, proved in this study to be a highly valuable tool for investigating dynamics of the upper troposphere even at high latitudes (up to $68^{\circ} \mathrm{N}$ ). Though a narrower WV channel would seem more fitted, the width of the METEOSAT WV channel did not appear as an insurmountable obstacle in the present study. However, a great improvement, which would preserve the advantage of a large bandpass channel for other study fields, would certainly be to lower the upper cut-off wavelength down to $7.1 \mu \mathrm{m}$ instead of the actual $7.4 \mu \mathrm{m}$, thus removing a narrow but rather inhomogeneously absorbing part of the channel band-pass.

Acknowledgments. We are deeply indebted to Mr. Foucault and the Service des Etudes Spéciales, Météorologie Nationale, who provided us with a copy of the radiosounding computer files needed in this study and to Jacqueline Duron for her most useful support in data processing. The constant encouragment of Pr. Morel is acknowledged hereby. We are grateful to the three anonymous referees and to Editor Dr. John J. Cahir, who reviewed this paper, for their critical suggestions and recommendations.

Part of this work was supported by C.N.R.S. (A.T.P. I.N.A.G. 78/3629); one of us acknowledges personal support (doctorate fellowship) provided by C.N.E.S.

\section{REFERENCES}

Danielsen, E. F., 1968: Stratospheric-tropospheric exchange based on radioactivity, ozone and potential vorticity. J. Atmos. Sci., 25, 502-518.

Goody, R. M., 1952: A statistical model for water vapour absorption. Quart, J. Roy. Meteor. Soc., 78, 165-169.

Martin, F. L., and V. V. Salomonson, 1970: Statistical characteristics of subtropical jet-stream features in terms of MRIR observations from Nimbus 2. J. Appl. Meteor., 9, 508-520.

Nordberg, W., A. W. McCulloch, L. L. Foshee, and W. R. Bandeen, 1966: Preliminary results from Nimbus 2. Bull. Amer. Meteor. Soc., 47, 857-872.

Palmen, E., and C. W. Newton, 1948: A study of the mean wind and temperature distribution in the vicinity of the polar front in winter. J. Meteor., 5, 220-226.

Paltridge, G. W., and C. M. R. Platt, 1976: Radiative Processes in Meteorology and Climatology. Elsevier, $318 \mathrm{pp}$.

Reiter, E. R., 1963: Jet Stream Meteorology. University of Chicago Press, $515 \mathrm{pp}$.

Rodgers, C. D., and C. D. Walshaw, 1966: The computation of infrared cooling rate in planetary atmospheres. Quart. J. Roy. Meteor. Soc., 92, 67-92.

Rodgers, E. B., V. V. Salomonson and H. L. Kyle, 1976: Upper tropospheric dynamics as reflected in Nimbus 4 THIR $6.7 \mu \mathrm{m}$ data. J. Geophys. Res., 81, 5749-5758.

Vuorela, L. A., 1957: A study of vertical velocity distribution in some jet-stream cases over Western Europe. Geophysica, 6, 68-90. 